\title{
Bond Yield Characteristics of Undisturbed Completely Decomposed Granite
}

\author{
Pan Liu, Xiaowen Zhou, and Yongbin He \\ School of Civil Engineering and Transportation, South China University of Technology, Guangzhou 510640, China \\ Correspondence should be addressed to Xiaowen Zhou; xwzhou616@163.com
}

Received 7 May 2015; Accepted 5 October 2015

Academic Editor: Santiago Garcia-Granda

Copyright ( 2015 Pan Liu et al. This is an open access article distributed under the Creative Commons Attribution License, which permits unrestricted use, distribution, and reproduction in any medium, provided the original work is properly cited.

\begin{abstract}
To investigate the bond yield characteristics of undisturbed completely decomposed granite (CDG) which is widely distributed in Southern China, a series of conventional drained tests were conducted. From the test data, some insights are obtained as follows: for undisturbed CDG soils, the bond yield point during shearing could be identified on the curve of the normalized tangential stiffness $E_{\text {tan }} / p^{\prime}$ versus axial strain on log-log scale. The consolidation pressure $P_{0}^{\prime}$ has a significant influence on the bond yield characteristics of undisturbed CDG during shearing, the bonds inside undisturbed CDG tend to be damaged relatively more seriously as $P_{0}^{\prime}$ is higher, accordingly the initial normalized stiffness $E_{\text {itan }} / p^{\prime}$ will be smaller at the beginning of the shearing process, and the axial strain at the bond yield point will also be smaller. Once consolidation pressure $P_{0}^{\prime}$ excesses a critical value such as $475 \mathrm{kPa}$ for this studied CDG, the bonds inside soil will be damaged totally at the consolidation compression stage. In this case, there will be no bond yield occurring during shearing and the $E_{\tan } / p^{\prime}-\varepsilon_{a}$ curves will coincide with the ones of reconstituted CDG soil. For reconstituted soils, no bond yielding was observed during shearing; the $E_{\tan } / p^{\prime}-\varepsilon_{a}$ curves are tightly consistent with no dependence of the value of $P_{0}^{\prime}$.
\end{abstract}

\section{Introduction}

In Hong Kong district and Guangdong province of China, about $63 \%$ of the urban areas are developed on granite [1]. These areas are subjected to subtropical climates such that considerable physical and chemical weathering of granite may take place. According to the Geotechnical Engineering Office (GEO) classification system (GEO 1988) [2], the granites are classified into 6 grades according to their weathering degrees, that is, fresh granite, slightly weathered granite, moderately weathered granite, strongly weathered granite, completely decomposed granite, and granite residual soil. For completely decomposed granite (CDG), it means the parent rock has been completely decomposed, but some of the original rock fabric and texture could be observed clearly. Besides, it can be easily crumbed by hand. The CDG soil is one of the most common geomaterials in Hong Kong district and Guangdong province [3]. Due to the lack of understanding of the fundamental behavior of CDG soils, there have been many failures of fill slopes during rainstorms, resulting in a substantial loss of lives and damage to property. These were the most common engineering disasters in these areas [4]. In addition, the development degree of the weathering crust is weather dependent. Geographic, geomorphic conditions and the environmental conditions result in strong regionalism and particularity of the CDG soil in these areas. It is urgent demand to research the mechanical behavior of the CDG soil in these areas.

Over the past decades, attempts have been made to investigate the mechanical behavior of the CDG soils [3-8]. It appears that most of these studies and their achievements concentrated on the physical and mechanical behaviors of reconstituted CDG soils. For instance, a series of triaxial tests, including isotropic and anisotropic compression, and drained and undrained shearing tests were conducted by Yan and $\mathrm{Li}[3]$ to understand the mechanical behavior of the CDG soils in Hong Kong. The undrained test results revealed that the overconsolidation ratio has a significant influence on the undrained behaviors. The stress paths of the overconsolidated specimens in $q-p^{\prime}$ plane are obviously different from the normally consolidated soils, but a unique state line can be identified in the triaxial space for the soil 
regardless of its initial state and stress path. To investigate the influence of stress ratio and stress path on the behaviors of loose composed granite, laboratory tests including undrained tests, drained tests with constant deviator stress, and a decreasing mean effective stress path were conducted by $\mathrm{Ng}$ et al. [4]. As these studies revealed, the isotropic compression lines (ICL) and critical state lines (CSL) are rather steady with no dependence of stress paths for CDG soils in their reconstituted states. In contrast, for undisturbed CDG soils, relevant researches were seldom reported. This may be due to the difficulty in in situ sampling of undisturbed CDG soils. Even so, it has been well known through engineering practices that the mechanical behaviors of undisturbed CDG are significantly different from its reconstituted state. Such differences may be aroused by the existent of the initial soil structure (fabric and bonds) inside undisturbed soil $[9,10]$. But up to now, the properties of the bonds and its effect on the mechanical behavior of undisturbed CDG are not very clear yet. In this paper, tests to compare undisturbed CDG and reconstituted CDG were performed to further investigate the effect of bonds on undisturbed CDG.

\section{Concept of Bond Yield of Soils}

Soil's initial structure is generally considered to be the source of differences in mechanical behavior of undisturbed soil and its remoulded counterpart. In the soil structure, the bonds of fabric connection among particles play an important role. Soil is a kind of material with nonlinear and elastoplastic properties. The yielding under stress action is an important behavior of soil. The stress yielding characteristics of structured soil should be extremely influenced by the initial bond (Malandraki and Toll, 2000 [11]). In this consideration, the term "bond yield" was adopted to explain the mechanical behaviors of structured soils in some literatures.

Anagnostopoulos et al. (1991) [12] defined a single yield point for Corinth marl based on the results of triaxial tests. The yielding point reflects a major loss of soil stiffness.

For artificially bonded soils, Malandraki and Toll (2000, 1996) $[11,13]$ defined yield from the tangential stiffness plotted against axial strain based on a series of triaxial drained and undrained tests as shown in Figure 1. The tangential stiffness $E_{\tan }$ can be calculated by the following formula according to the elasticity theory:

$$
E_{\tan }=\frac{\left(\delta \sigma_{1}^{\prime}-2 \nu \sigma_{3}^{\prime}\right)}{\delta \varepsilon_{a}},
$$

where $\sigma_{1}^{\prime}$ is the maximum principal effective stress, $\sigma_{3}^{\prime}$ is the minimum principal effective stress, $v$ is the Poisson ratio, and $\varepsilon_{a}$ is the axial strain during shearing.

As seen in Figure 1, three yield points are defined. The first yield point reflects a primary obvious loss in stiffness. After the first yield, however, the soil remains relatively high stiffness with the development of strain until an abrupt drop occurs. This second yield is defined as bond yield. The final yield is defined as the point of maximum curvature of the stress-strain curve plotted on natural scale. The final yield means that the bonded soil has lost all of its stiffness due

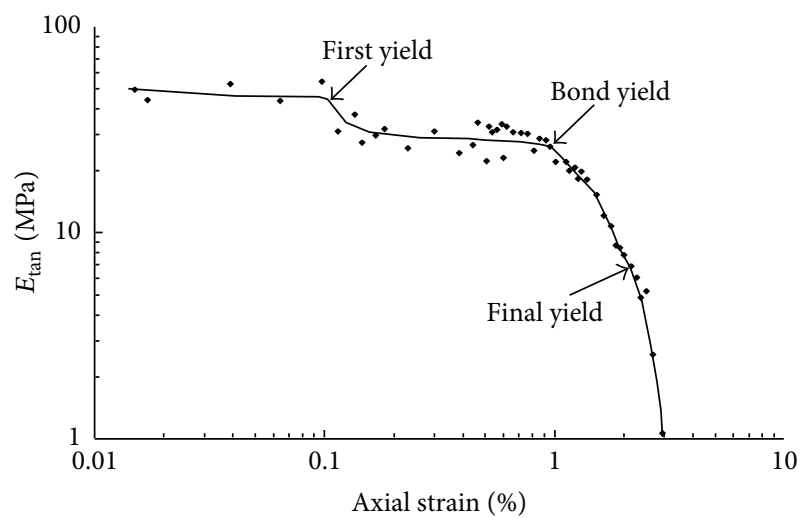

FIGURE 1: Definition of the three yield conditions for bonded soils under shearing (Malandraki and Toll, 2000) [11].

to bonding. For the three yield points, Malandraki and Toll $(2000,1996)[11,13]$ thought that the bond yield point is rather important because it reveals the inherent information about the bond and can better clarify the behavior of artificially bonded soils under shearing.

As for the undisturbed CDG studied in this paper, different from artificially bonded soils, the bonds in natural soil are heterogeneous. So the intrinsic small strain behavior could not be captured well and the first yield points are not always well defined. In this paper, the bond yield which related to the soil structure collapse was focused on, and the final yield which means that the bonded soil has lost all of its stiffness due to bonding was not discussed here.

The influence of consolidation pressure $P_{0}^{\prime}$ on bond yield characteristics of undisturbed CDG was researched in this study with a series of conventional triaxial drained tests conducted.

\section{Description of Soil Samples}

The completely decomposed granite soil used in this study was taken from the \#D1 construction site located at the Zhonghang Mall of Futian District, Shenzhen city, China. The authority who issued the permission for soil sampling is the Shenzhen branch of China Academy of Railway Sciences. According to the geological report, the CDG is the result of weathering of the Late Yanshanian granite. In order to minimize the disturbance of sampling, large square block soil samples were extracted from the excavated foundation pit about $11 \mathrm{~m}$ below the ground surface as shown in Figure 2. By visual inspection, the soil, in mainly flesh red with some brownish yellow, celadon, and off-white speckles, is a medium-grained CDG soil with substantial amount of clays and silts.

In order to obtain the physical properties of the soil, the free-fall method proposed by the Japanese Society of Soil Mechanics and Foundation Engineering (JSSMFE, 1982) [14] was adopted to ensure that small lumps of soil specimens were broken up consistently. In this method, firstly, the CDG was oven-dried for 24 hours. Then each of $500 \mathrm{~g}$ soils contained in plastic bags was allowed to fall freely onto a concrete floor 


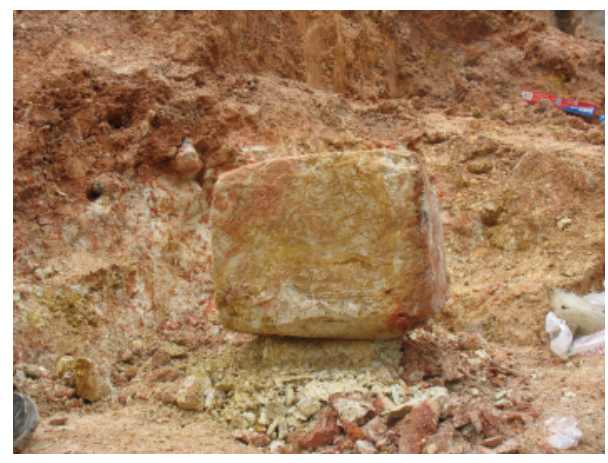

FIgURE 2: Soil sample at the construction site.

from $1.5 \mathrm{~m}$ height for 30 times. After the above treatments, dry sieving was performed and particles larger than $5 \mathrm{~mm}$ were discarded (about $0.4 \%$ by weight).

By following the test procedures given in BS1377 (British Standards Institution, 1990) [15], sieving method and hydrometer method were used to get the particle size distribution (PSD) curve of this CDG. Soil sample was firstly wet-sieved prior to any treatment. Then the soil retained on the $63 \mu \mathrm{m}$ sieve was oven-dried and then sieved with multigrades openings. The soil under the $63 \mu \mathrm{m}$ sieve was analyzed by hydrometer method (a sedimentation test). Such steps were thought to be capable of minimizing the crushing of coarse particles [16] and an accurate full-gradation curve could be obtained as shown in Figure 3. It can be seen that the soil used in this study covers a large range of particle sizes. The soil comprises $11 \%$ gravel, $45 \%$ sand, $35 \%$ silt, and $9 \%$ clay, which can be classified as a medium-grained soil. Besides, the average particle size is $0.1 \mathrm{~mm}$.

Particle size distribution curves obtained by other researchers [16-19] for some other soils are also shown in Figure 3 for comparison. Similar to these researches, the soil in this study is well graded. Yet the studied soil has the mean grain size $0.1 \mathrm{~mm}$ smaller than others and shows more fine particles. This may be due to its environment which is more favorable to the chemical weathering. This difference of gradation may result in differences of mechanical behavior.

The wet density of the undisturbed soil is $1700 \mathrm{~kg} / \mathrm{m}^{3}$ and the moisture content is $19.35 \%$. The specific gravity of the soil was found to be 2.60. From the Standard Proctor Compaction Tests, the maximum dry density of the soil is $1650 \mathrm{~kg} / \mathrm{m}^{3}$ with the optimum moisture content at $22 \%$. The plastic limit and liquid limit are $25.2 \%$ and $40 \%$, respectively, and the plastic index is $14.8 \%$.

\section{Specimen Preparation and Triaxial Test Procedures}

For triaxial tests, undisturbed specimens of $39.1 \mathrm{~mm}$ in diameter and $80 \mathrm{~mm}$ in height were hand-trimmed from the block samples extracted from the construction site. Careful actions were taken to ensure that the soil specimens were not disturbed. The reconstituted specimens were prepared by the wet tamping method (dynamic compaction). The specimens

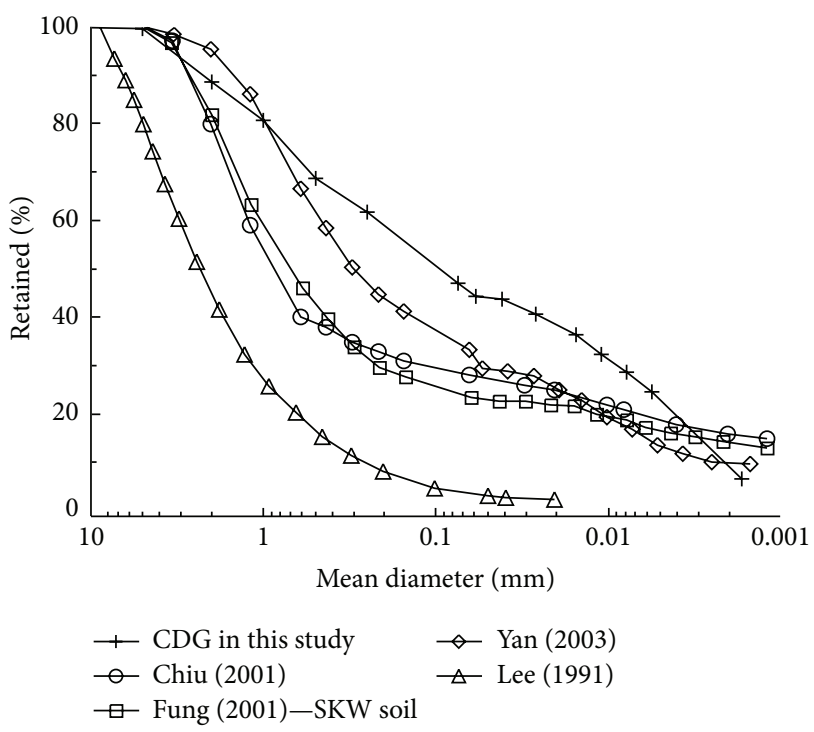

FIgURE 3: Particle size distribution of CDG.

were compacted inside a split mold in 5 layers, with scratching between each layer. The initial dry density of reconstituted soil specimens was equal to the undisturbed specimens.

To make triaxial specimens saturated before test, the following saturation procedures were implemented. Firstly prepared specimen was installed into a computer-controlled Bishop \& Wesley triaxial stress path cell; then the specimen was preliminary saturated by flushing it with carbon dioxide for 45 minutes and then flushing with deaired water for 2 hours. After that treatment, $200 \mathrm{kPa}$ of back pressure was applied in order to increase the degree of saturation in the specimen. During this process, the cell pressure should be increased simultaneously with the back pressure to make sure that the effective confining pressure is unchanged. The loading rate of back pressure and cell pressure was $50 \mathrm{kPa} / \mathrm{h}$. After the above treatments, the Skempton pore-pressure parameter ( $B$ value) of each specimen was measured higher than 0.97 .

After saturation, a series of consolidated drained tests (CD Tests) were conducted. Specimens were isotropically consolidated at different confining pressures with the loading rate of $15 \mathrm{kPa} / \mathrm{h}$ and then were sheared under drained condition. In the shearing process, the axial strain rate was controlled at $0.02 \% / \mathrm{min}$. The axial strains were measured using the internal devices LVDTs. The measured effective stress paths in this study are plotted in Figure 4.

In Figure 4, the specimen names indicate the consolidation pressure and whether it is undisturbed soil or not. For example, CD125 means an undisturbed soil specimen consolidated at $P_{0}^{\prime}=125 \mathrm{kpa}$; specimen CD75r means a reconstituted soil specimen consolidated at $P_{0}^{\prime}=75 \mathrm{kpa}$.

\section{Test Results}

Figures 5 and 6 show the typical stress-strain relationship and the volumetric-axial strain relationship of the drained tests on 


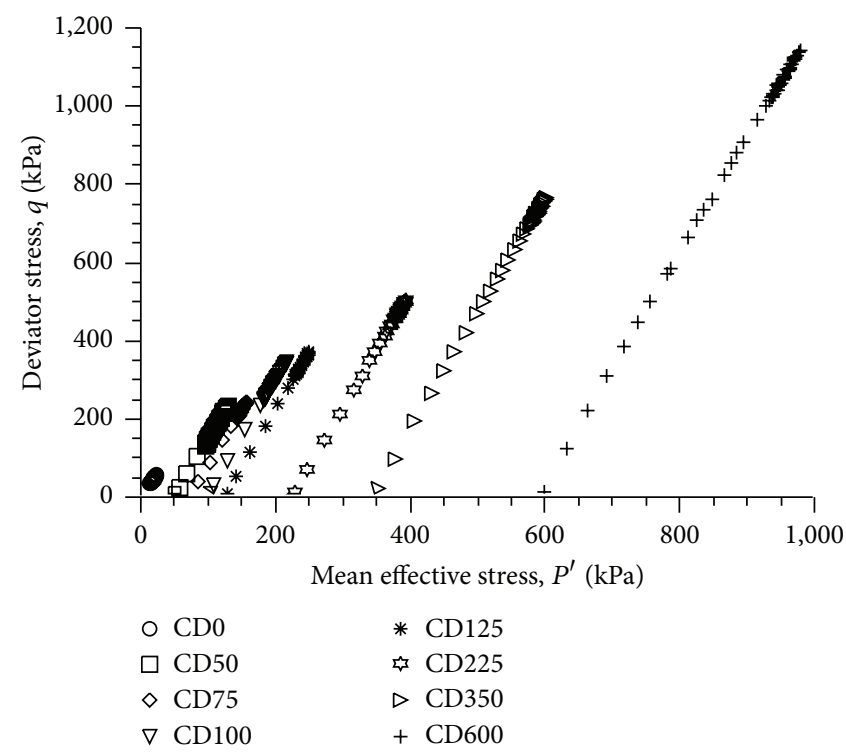

(a) Stress paths of undisturbed CDG

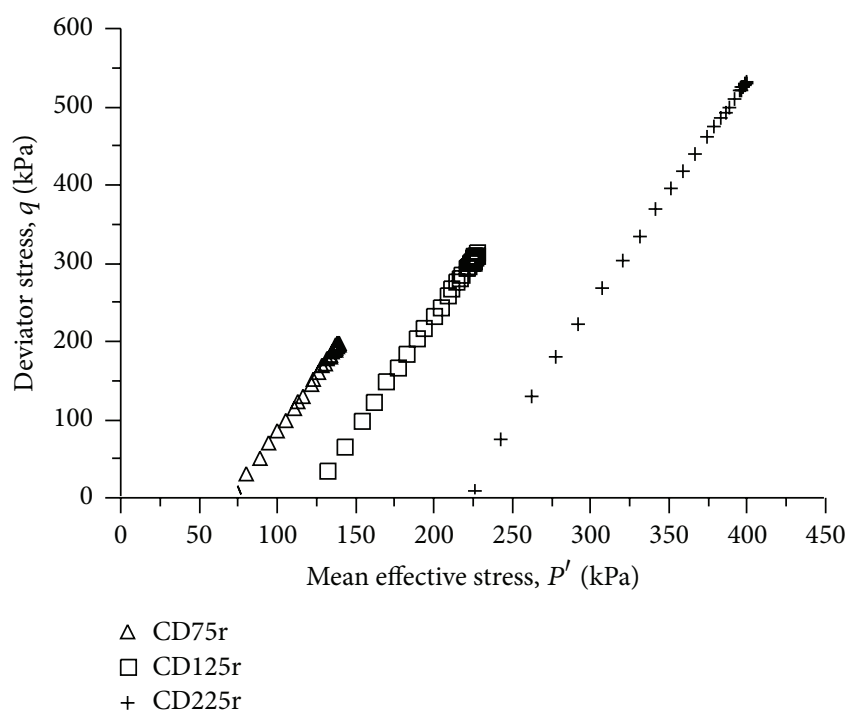

(b) Stress paths of reconstituted CDG

FIGURE 4: Stress paths of consolidated drained tests.

the undisturbed specimens and the reconstituted specimens, respectively.

As shown in Figure 5(a), strain softening is observed in all the undisturbed specimens. The axial strain at peak deviator stress becomes larger as the consolidation pressure increases. After a peak reached, the deviator stress decreases with the development of axial strain. The volumetric-axial strain relationship of the undisturbed specimens is shown in Figure 5(b), from which the tests CD0, CD50, CD75, CD100, and CD125 show contractive behavior at the beginning of shearing $\left(\varepsilon_{a}<2 \%\right)$ and then turn to expansive behavior with the development of axial strain. Tests CD225, CD350, and CD600 show allover contractive behavior during shearing. Figure 6 reveals that all the reconstituted specimens show contractive behavior and strain-hardening response regardless of the different consolidation pressures.

\section{Bond Yield of Undisturbed CDG}

Since $\delta \sigma_{3}^{\prime}=0$ during the shearing process of consolidated drained tests in this study, the tangential stiffness $E_{\tan }$ can be calculated by the following formula from (1):

$$
E_{\tan }=\frac{\delta \sigma_{1}^{\prime}}{\delta \varepsilon_{a}} .
$$

The tangential stiffness values should be normalized by the current value of mean effective stress $p^{\prime}$ because the stiffness values are related to the stress level [11, 20]. Such normalization can remove the effect of stress level during shearing and so the stiffness values of different tests can be compared with each other. The normalized tangential stiffness $E_{\tan } / p^{\prime}$ for tests CD75, CD125, and CD225 and CD75r, CD125r, and CD225r is shown in Figure 7 plotted versus axial strain on a log-log scale.
For the reconstituted specimens (CD75r, CD125r, and CD225r), as shown in Figure 7 , the $E_{\text {tan }} / p^{\prime}-\varepsilon_{a}$ curves decrease gradually with no abrupt drop occurrence. Besides, the three curves appear to be very close to each other like a single line. This means that the consolidation pressure $P_{0}^{\prime}$ has no significant influences on the normalized tangential stiffness. This indicates that the $E_{\tan } / p^{\prime}-\varepsilon_{a}$ curve of reconstituted CDG may be invariable and independent of the consolidation pressure $P_{0}^{\prime}$.

However, the development patterns of curves for undisturbed specimens (CD75, CD125, and CD225) are obviously different from reconstituted specimens. For each curve of the undisturbed specimens, there is an abrupt drop occurring at a certain strain. Besides, these curves are not close to each other for different consolidation pressures. The normalized stiffness $E_{\mathrm{tan}} / p^{\prime}$ of undisturbed specimens (e.g., CD75) takes a relatively high value until the abrupt drop occurs; after that, the stiffness decreases very quickly than reconstituted specimens to the values even lower than those of the latter. Here, the key points at which the abrupt drops occur are thought to achieve the state of bond yield.

In order to compare the effect of different consolidation pressures $P_{0}^{\prime}$ on bond yield characteristics of undisturbed specimens, the results of tests CD0, CD50, CD100, CD225, CD350, and CD600 are plotted together in Figure 8. The curves of reconstituted specimens in Figure 7 are represented by a single line which is also plotted in Figure 8 . As shown in Figure 8, the initial normalized tangential stiffness value $E_{\text {itan }} / p^{\prime}$ of test CD0 is about 250 obviously higher than others at the beginning of the shearing process, and the value of CD350 is only about 80 slightly higher than that of reconstituted specimen. As the consolidation pressure reaches $600 \mathrm{kPa}$, the initial value of CD600 is almost equal to that of reconstituted CDG soil. So, the lower the consolidation pressure $P_{0}^{\prime}$ is, the higher the initial normalized stiffness 


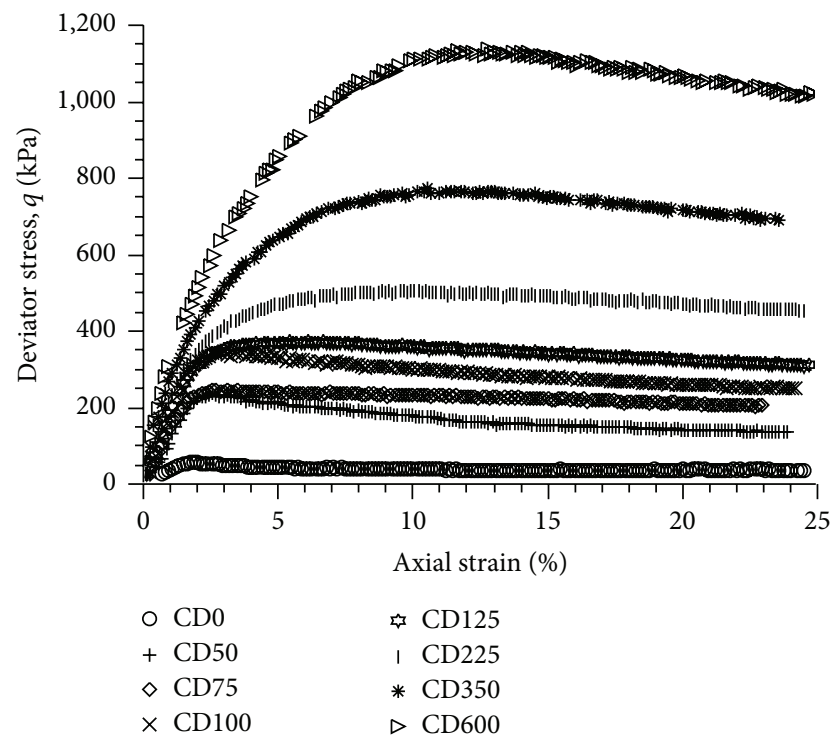

(a) Stress-strain relationship

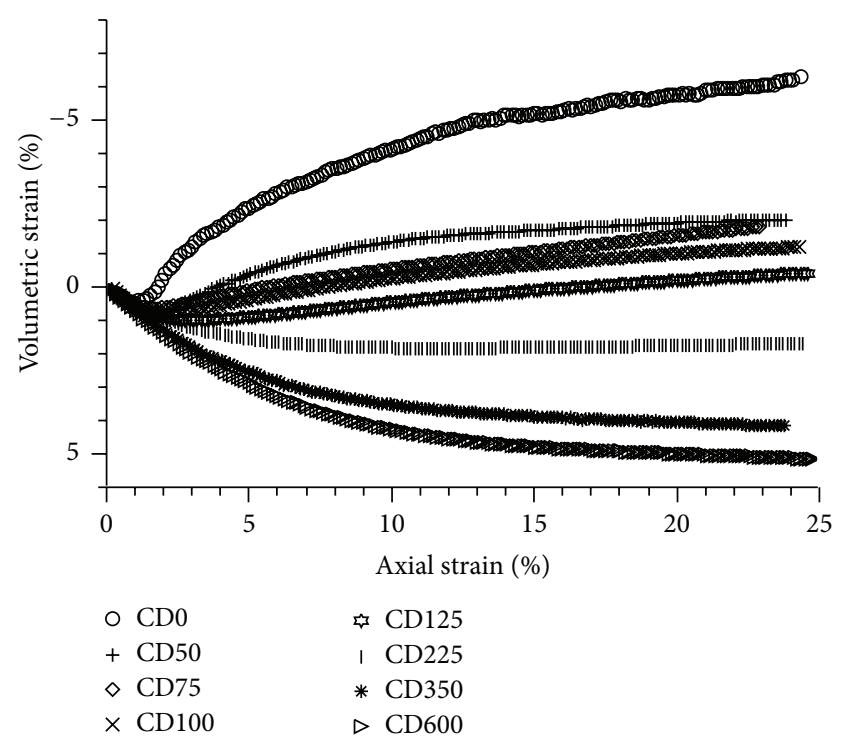

(b) Volumetric-strain relationship

FIGURE 5: Undisturbed CDG curves.

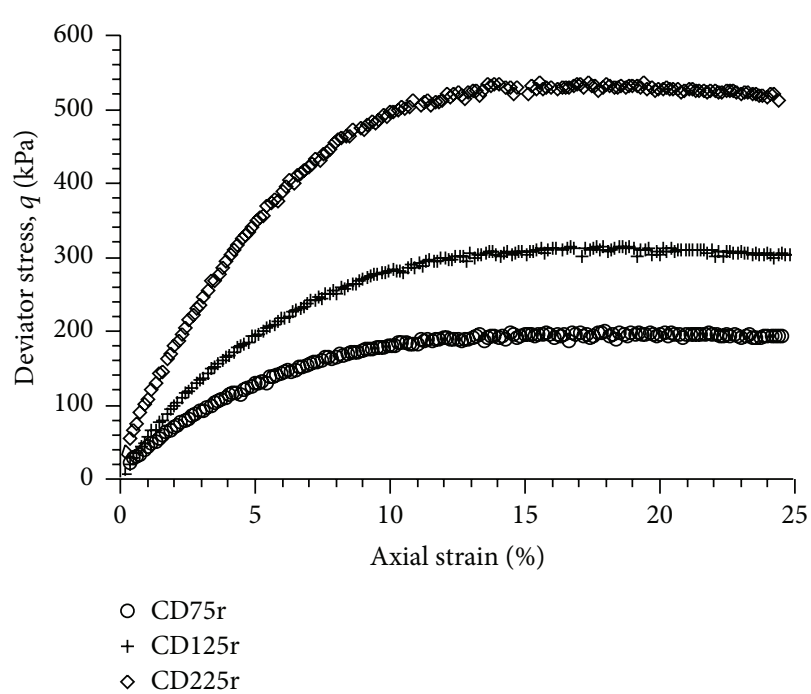

(a) Stress-strain relationship

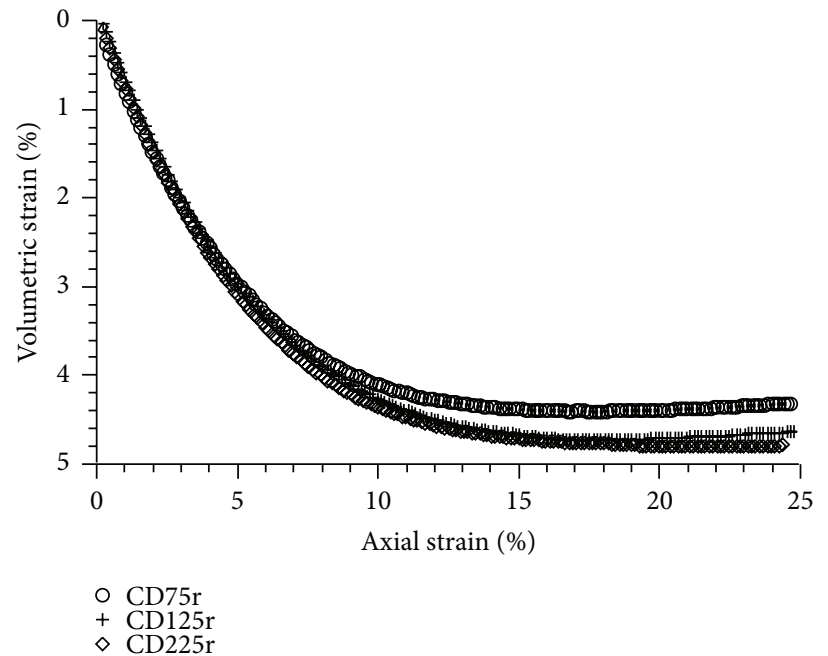

(b) Volumetric-strain relationship

FIgURE 6: Reconstituted CDG curves.

for undisturbed CDG is. According to above analysis, the internal bond structure of undisturbed CDG soil should be the main reason that results in the significant increase of the normalized stiffness especially at a lower consolidation pressure level. But as consolidation pressure gets great enough, the effect of bond structure will be eliminated totally in the consolidation stage. The internal structure should be induced or dominated by some kinds of bond fabric action among soil particles. The structural (bond) effect will be destroyed remarkably as strain rise to a certain value, which corresponds to the state of bond yield with an abrupt drop of normalized stiffness. In a word, the consolidation pressure $P_{0}^{\prime}$ directly influences the behavior of undisturbed CDG soil especially at low consolidation pressure.
For the bond yield point, as seen in Figure 8, the higher the consolidation pressure $P_{0}^{\prime}$ is, the corresponding axial strain is smaller. The bond yield occurs at $\varepsilon_{a}=1.53 \%$ for CD0 and $1.28 \%, 1.15 \%, 0.75 \%$, and $0.41 \%$ for CD50, CD100, CD225, and CD350, respectively. After the bond yield, the normalized stiffness decreases very quickly with the strain development. However, there is no bond yield for CD600. The curve of CD600 is almost close to the reconstituted soil curve.

\section{Summary of Insights}

Burland (1990) [9] and Yan and Li (2011) [8] reported that the bonds inside undisturbed soil would be damaged by compression and shear actions. It means that the two stages 


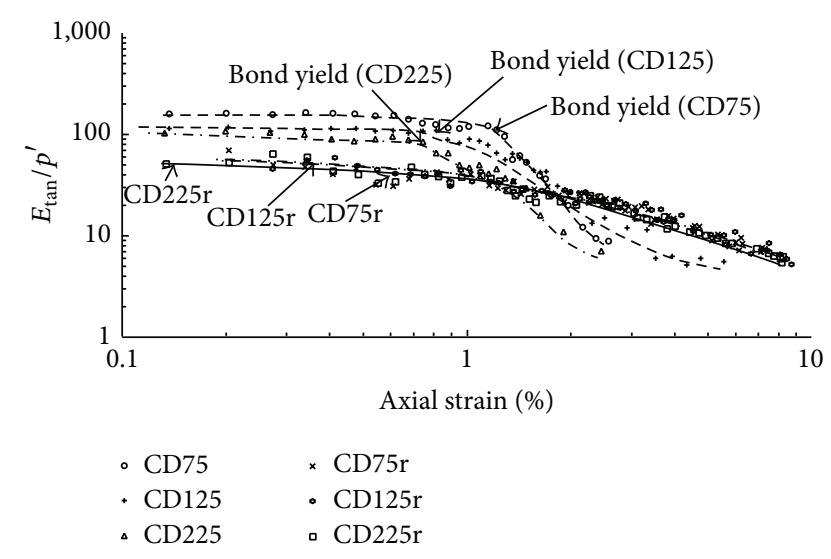

FIGURE 7: Bond yield for undisturbed and reconstituted specimens at the same $P_{0}^{\prime}(75,125$, and $225 \mathrm{kPa})$.

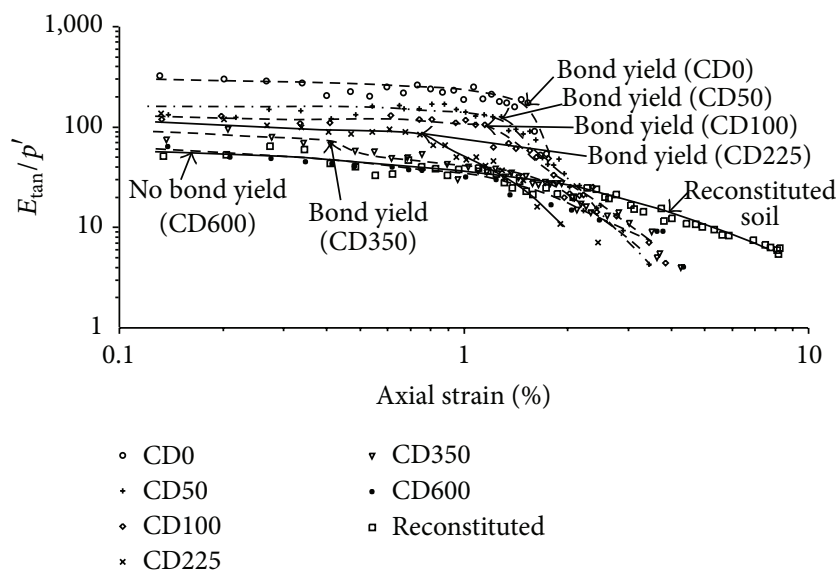

FIGURE 8: Bond yield for undisturbed specimens at $P_{0}^{\prime}$ ranged within $0 \mathrm{kPa} \sim 600 \mathrm{kPa}$.

of isotropic consolidation and shear (just axial loading) will lead to damage of soil's bond structure. The test results in this study provide further evidences for this viewpoint and some new insights are obtained and summarized below.

(i) The greater the consolidation pressure $P_{0}^{\prime}$ is, the more seriously the initial bond structure is damaged. As no consolidation pressure $\left(P_{0}^{\prime}=0 \mathrm{kpa}\right)$ was applied on an undisturbed specimen, the bonds inside specimen should be intact before shearing. So the initial normalized tangential stiffness $E_{\text {itan }} / p^{\prime}$ should be the highest due to the strongest effect of intact bonds remaining in the specimen. The tests indeed gave the anticipated results. Specimen CD0 has the highest value of 250 for initial normalized stiffness. The initial normalized stiffness $E_{\text {itan }} / p^{\prime}$ decreases with the increase of consolidation pressure $P_{0}^{\prime}$. As $P_{0}^{\prime}$ is great enough such as $600 \mathrm{kPa}$, the initial bond structure may be damaged totally by consolidation compression action. Hence it is easy to understand that the initial normalized stiffness of CD600 has almost the same value as that of reconstituted specimen.

(ii) During the shearing process, the bond structure should be damaged gradually and may encounter abrupt loss at some certain strain level. This kind of mechanism

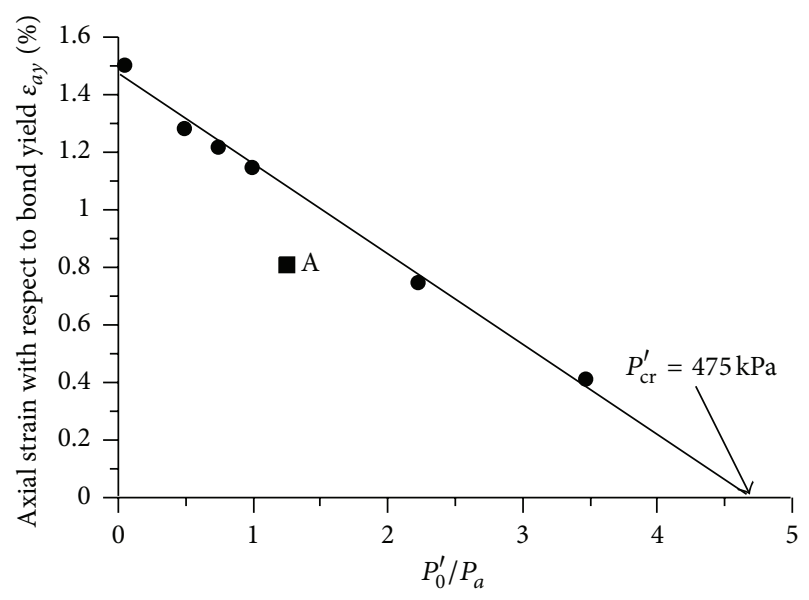

FIGURE 9: Relationship between $\varepsilon_{a y}$ and $P_{0}^{\prime} / P_{a}$.

is demonstrated very well by the results as shown in Figure 8 . In Figure 8, regarding the undisturbed specimens, we can see that the normalized tangential stiffness decrease gradually at low strains but suddenly drop down at some certain strains. These processes of weakening of soils reflect the damage of bond structure induced by shearing force. Moreover, the transitional points on these $E_{\tan } / p^{\prime}-\varepsilon_{a}$ curves where stiffness undergoes an abrupt reduction indicate the existing of bond yield in undisturbed CDG soils.

(iii) Here we denote $\varepsilon_{a y}$ as the axial strain at which bond yield takes place with stiffness abrupt drops. Figure 9 shows the scattered points $\left(P_{0}^{\prime} / P_{a}, \varepsilon_{a y}\right)$ of each undisturbed specimen, where $P_{a}$ is the atmospheric pressure. It can be seen that these scattered points comply well with a linear relationship except point A (test CD125). Point A should be regarded as an outline point due to test error. The fitted line is described by the following equation:

$$
\varepsilon_{a y}=1.47-0.3144 \frac{P_{0}^{\prime}}{P_{a}} \quad\left(R^{2}=0.9919\right) .
$$

In (3), let $\varepsilon_{a y}=0$; get $P_{0}^{\prime}=475 \mathrm{kpa}$. This indicates that as consolidation pressure is great enough as $P_{0}^{\prime}=475 \mathrm{kpa}$, the initial bond structure will be totally damaged at the stage of consolidation compression, so that there is no abrupt reduction of stiffness during the shearing process. In this case, the behavior of the undisturbed specimen is similar with the reconstituted soil (no bond yield occurring) during shearing. In other words, this indicates that the influence of bonds would be removed before shearing as $P_{0}^{\prime} \geq 475 \mathrm{kpa}$ and the behavior of the undisturbed soils would be similar with reconstituted soils during shearing, such as $\mathrm{CD} 600$, as shown in Figure 8.

(iv) Based on the results shown in Figure 7, for reconstituted CDG soils, the curves of $E_{\tan } / p^{\prime}-\varepsilon_{a}$ tend to be distributed very closely which can be approximately represented by a single line as shown in Figure 8. It means there is no bond structure existing in reconstituted soil. The value of $P_{0}^{\prime}$ could only change the stress level before shearing, and its effect on tangential stiffness can be removed by 
the normalization of the current value of mean effective stress $p^{\prime}$. In a word, the $E_{\tan } / p^{\prime}-\varepsilon_{a}$ curves of reconstituted soils have the feature of uniqueness which decreases gradually with strain development. It may be an intrinsic characteristic of the reconstituted CDG soil studied in this paper.

\section{Conclusions}

Based on the test results and analysis, the following conclusions can be obtained:

(i) The existing of initial bond structure may significantly influence the mechanical behavior of undisturbed completely decomposed granite especially under low confining (consolidation) pressure.

(ii) In the consolidation stage, the higher the confining pressure is, the more seriously the bonds are damaged. As the confining pressure is up to a certain value, such as $475 \mathrm{kPa}$ for the CDG in this study, the effect of bond structure may be totally eliminated by the compression action.

(iii) In the shearing stage, the normalized tangential stiffness of undisturbed CDG decreases gradually with the axial strain development. As the strain reaches a certain value, the bond structure will get yielding; then the stiffness will appear to be much smaller with the strain development.

(iv) The strain of bond yield is linearly related to the consolidation confining pressure.

\section{Conflict of Interests}

The authors declare that there is no conflict of interests regarding the publication of this paper.

\section{Authors' Contribution}

Pan Liu conceived, designed, and performed the experiments. Xiaowen Zhou and Pan Liu analyzed the data. Yongbin He and Pan Liu wrote the paper.

\section{Acknowledgments}

The authors are grateful to the support by the National Natural Science Foundation of China (no. 51109083); the research grant provided by the State Key Lab of Subtropical Building Science, South China University of Technology (no. 2013ZC04); the Water Conservancy Science and Technology Innovation Project of Guangdong Province (no. 2015-17); and the research grant provided by Guangzhou Forestry and Garden Administrative Bureau (no. 2012-36).

\section{References}

[1] R. Shaw, "Variations in sub-tropical deep weathering profiles over the Kowloon granite, Hong Kong," Journal of the Geological Society, vol. 154, no. 6, pp. 1077-1085, 1997.
[2] Geotechnical Engineering Office (GEO), Guide to Rock and Soil Descriptions, Geoguide 3, Geotechnical Engineering Office (GEO), Hong Kong, 1988.

[3] W. M. Yan and X. S. Li, "Mechanical response of a mediumfine-grained decomposed granite in Hong Kong," Engineering Geology, vol. 129-130, pp. 1-8, 2012.

[4] C. W. W. Ng, W. T. Fung, C. Y. Cheuk, and L. Zhang, "Influence of stress ratio and stress path on behavior of loose decomposed granite," Journal of Geotechnical and Geoenvironmental Engineering, vol. 130, no. 1, pp. 36-44, 2004.

[5] I. K. Lee and M. R. Coop, "The intrinsic behaviour of a decomposed granite soil," Geotechnique, vol. 45, no. 1, pp. 117-130, 1995.

[6] C. W. W. Ng and A. C. F. Chiu, "Laboratory study of loose saturated and unsaturated decomposed granitic soil," Journal of Geotechnical and Geoenvironmental Engineering, vol. 129, no. 6, pp. 550-559, 2003.

[7] Y. H. Wang and W. M. Yan, "Laboratory studies of two common saprolitic soils in Hong Kong," Journal of Geotechnical and Geoenvironmental Engineering, vol. 132, no. 7, pp. 923-930, 2006.

[8] W. M. Yan and X. S. Li, "A model for natural soil with bonds," Geotechnique, vol. 61, no. 2, pp. 95-106, 2011.

[9] J. B. Burland, "On the compressibility and shear strength of natural clays," Géotechnique, vol. 40, no. 3, pp. 329-378, 1990.

[10] S. Leroueil and P. R. Vaughan, "The general and congruent effects of structure in natural soils and weak rocks," Geotechnique, vol. 40, no. 3, pp. 467-488, 1990.

[11] V. Malandraki and D. G. Toll, "Drained probing triaxial tests on a weakly bonded artificial soil," Géotechnique, vol. 50, no. 2, pp. 141-151, 2000.

[12] A. G. Anagnostopoulos, N. Kalteziotis, G. K. Tsiambaos, and M. Kavvadas, "Geotechnical properties of the Corinth Canal marls," Geotechnical and Geological Engineering, vol. 9, no. 1, pp. $1-26,1991$.

[13] V. Malandraki and D. G. Toll, “The definition of yield for bonded materials," Geotechnical and Geological Engineering, vol. 14, no. 1, pp. 67-82, 1996.

[14] Japanese Society of Soil Mechanics and Foundation Engineering (JSSMFE), Soil Testing Methods, Japanese Society of Soil Mechanics and Foundation Engineering (JSSMFE), Tokyo, Japan, 2nd edition, 1982.

[15] British Standards Institution (BSI), "Methods of tests for soils for civil engineering purposes," BS 1377, British Standards Institution (BSI), London, UK, 1990.

[16] W. M. Yan, Experimental study and constitutive modelling of recompacted completely decomposed granite [Ph.D. dissertation], Hong Kong University of Science and Technology, Hong Kong, 2003.

[17] C. F. Chiu, Behaviour of unsaturated loosely compacted weathered materials [Ph.D. thesis], Hong Kong University of Science and Technology, Hong Kong, 2001.

[18] W. T. Fung, Experimental study and centrifuge modelling of loose fill slope [M.Phil. dissertation], Hong Kong University of Science and Technology, Hong Kong, 2001.

[19] I. K. Lee, Mechanical behaviour of compacted decomposed granite soil [Ph.D. Dissertation], City University London, London, UK, 1991.

[20] V. Malandraki and D. G. Toll, "Triaxial tests on weakly bonded soil with changes in stress path," Journal of Geotechnical and Geoenvironmental Engineering, vol. 127, no. 3, pp. 282-291, 2001. 

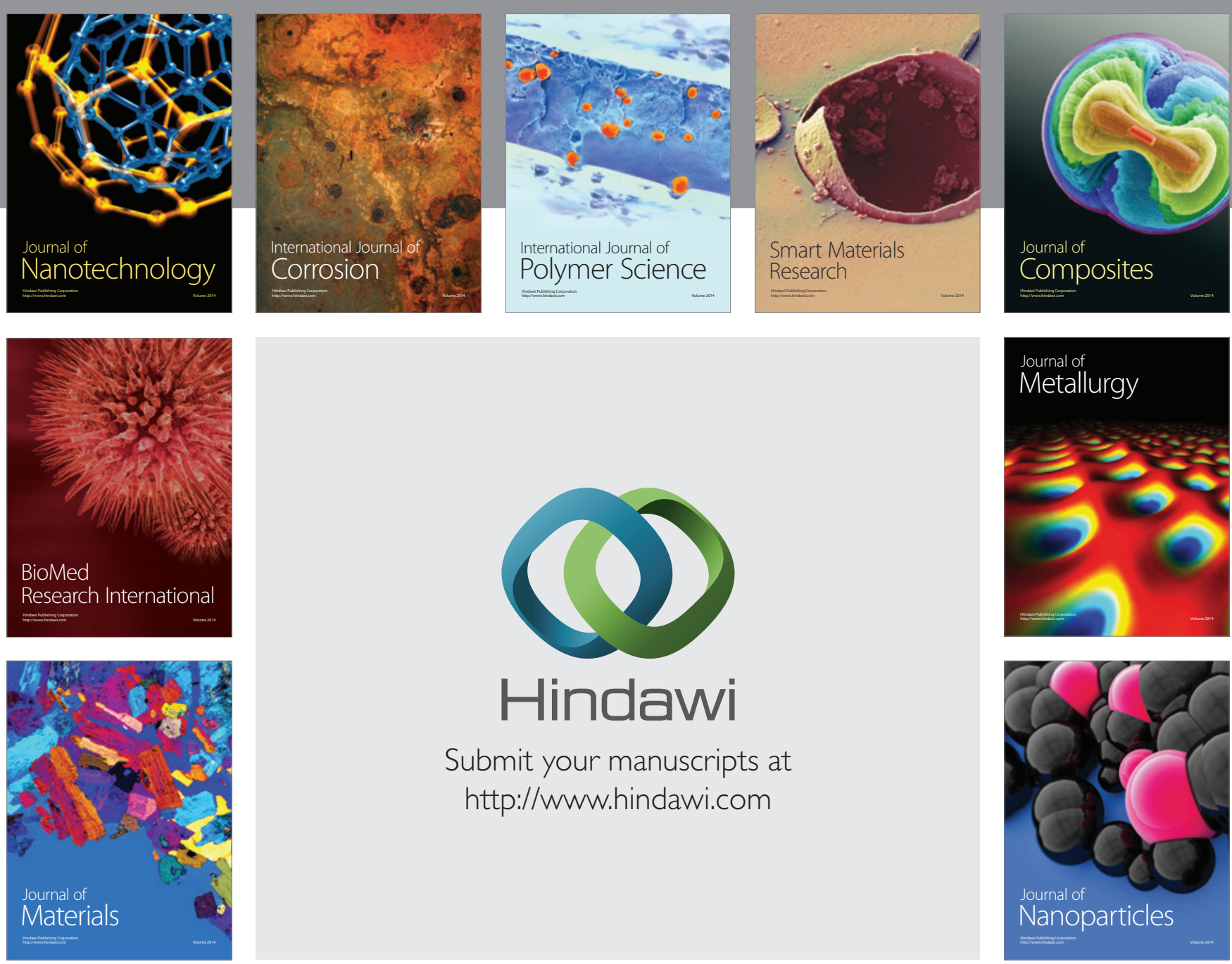

Submit your manuscripts at http://www.hindawi.com
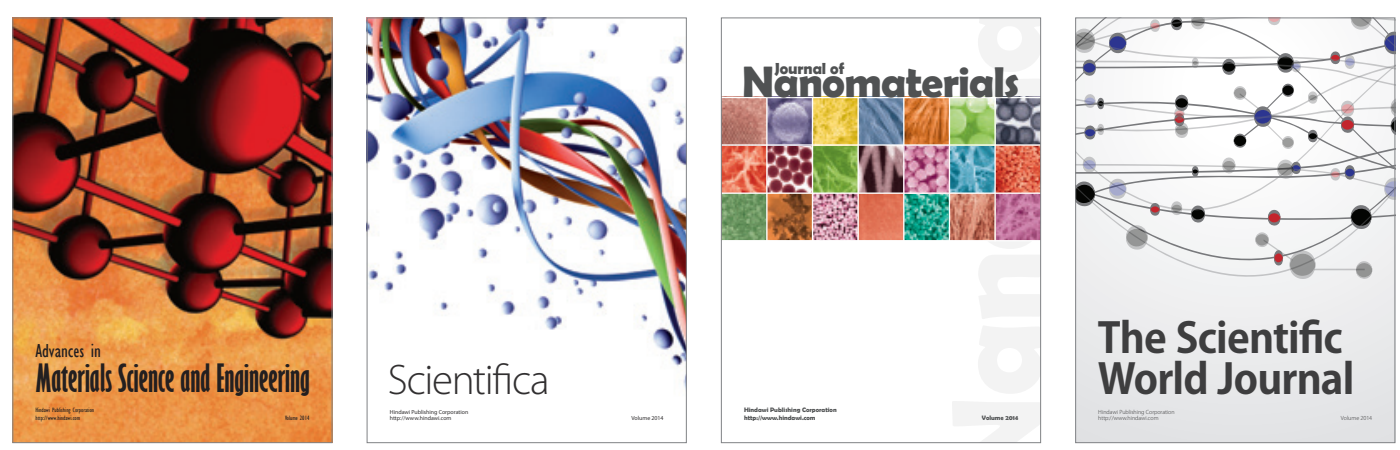

\section{The Scientific World Journal}
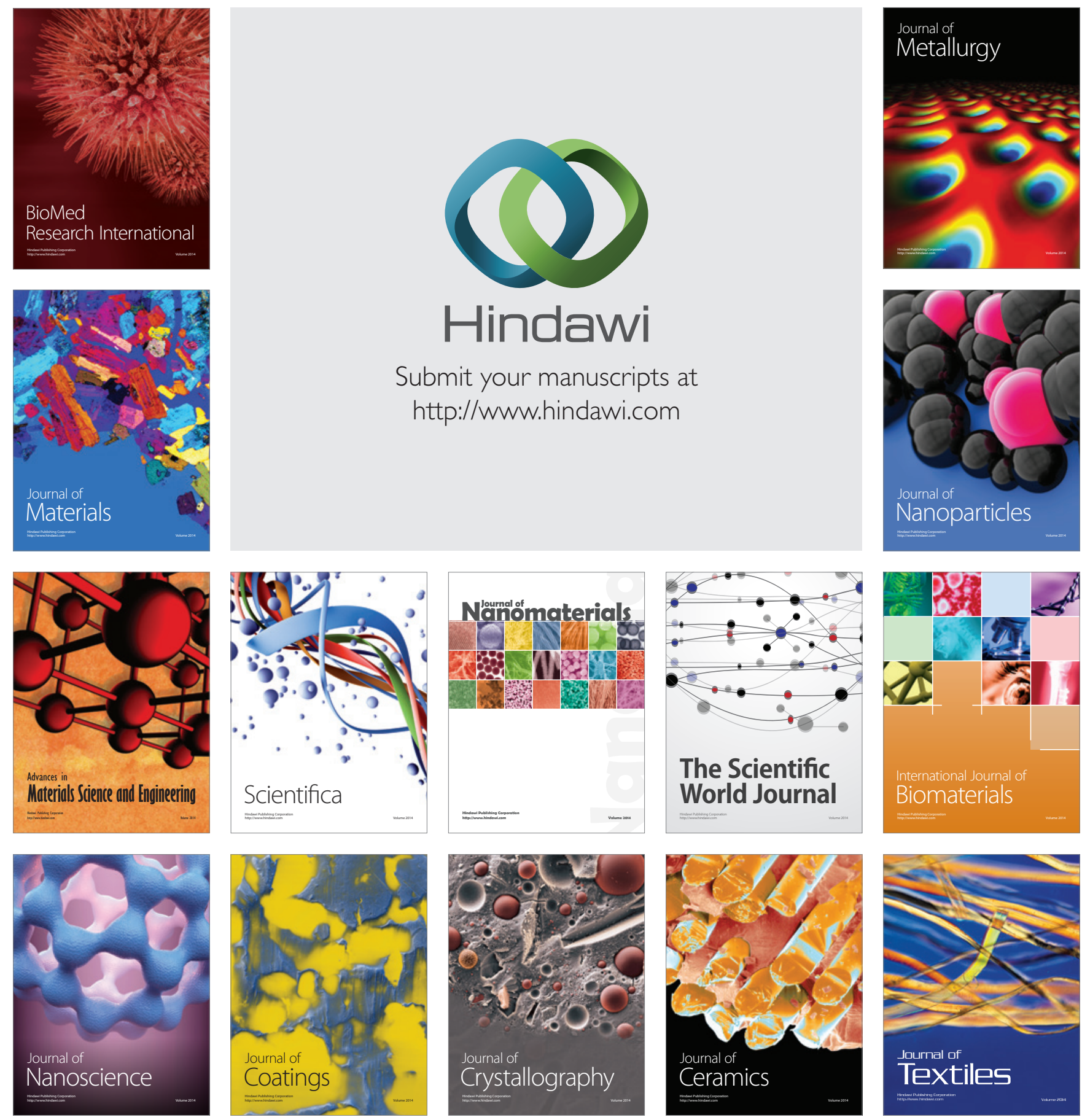